BLS 35, No 1 2009. DOI: http://dx.doi.org/10.3765/bls.v35i1.3622

(published by the Berkeley Linguistics Society and the Linguistic Society of America)

\title{
Mandarin Vowels Revisited: Evidence from Electromagnetic Articulography
}

\author{
CHEN-HUEI WU and CHILIN SHIH \\ University of Illinois, Urbana-Champaign
}

\section{Introduction}

The distributional properties of Mandarin vowels provide an excellent opportunity to examine issues related to the categorization of speech sounds. Structural analysis of Mandarin vowels has been done in a number of phonological theories (Cheng 1973, Lin 1989, Wang 1993, Duanmu 2000). Acoustic properties and articulatory movements are both reported in phonetic studies (Howie 1970, Shih 1995, Torng 2000). Previous studies bring us to deeper questions about the reality of Mandarin vowels. How do native speakers produce vowels in context? How are vowels in Mandarin categorized? In this study, the goal is to demonstrate phonological variations of Mandarin vowels in terms of articulatory and acoustic properties with Electromagnetic Articulography AG500 (hereafter EMA). By studying Mandarin vowels, we stand to gain a better understanding of how they are categorized in terms of articulatory and acoustic properties.

In the following, the Mandarin phonetic system will be introduced in Section 1. The previous studies of Mandarin vowels related to the transcription systems, phonological categories, phonetic measurements, as well as the articulatory investigations will be reviewed in Section 2. The methodology will be described in Section 3. The data analysis and conclusion will be presented in Section 4 and Section 5, respectively.

\section{The Phonetic System of Mandarin}

The Mandarin vowel inventory is a controversial topic. Many vowels occur in complementary distribution. Without minimal pairs, it is not a straightforward task to provide proofs of vowel categories in either the phonological input or the phonological output. As a starter, Table 1 lists 12 monophthongs in Mandarin, including a retroflex vowel. These vowels are classified in three dimensions, namely: [high/low], [front/back], [rounded/unrounded]. However, Table 1 is far from being a consensus view. There is little agreement in the literature. We do not know the maximum number of Mandarin vowels, though the number of possible 


\section{Chen-huei Wu and Chilin Shih}

rhymes is generally agreed on; we do not know whether native Mandarin speakers and listeners treat some of these vowels as the same, and if so, which ones should be combined, and how similarity is measured.

Table 1. Vowels in Mandarin

\begin{tabular}{|c|c|c|c|c|c|c|c|}
\hline & \multicolumn{2}{|c|}{ FRONT } & & NTRAL & \multicolumn{3}{|c|}{ BACK } \\
\hline HIGH & & $\mathrm{y}$ & $\dot{\mathrm{t}}$ & & & $\mathrm{u}$ & $\mathrm{u}$ \\
\hline MID & e & & $\partial$ & $\gamma^{2}$ & $(\gamma)$ & & \\
\hline LOW & $\mathrm{a}$ & & & & & a & \\
\hline
\end{tabular}

In Mandarin, the possible syllable structures are CGVN, CGVG, CGV, CVN, CVG, GVN, GVG, CV, GV, VC, VG, V. ${ }^{1}$ Only alveolar or velar nasals [n, y] and glides $[\mathrm{j}, \mathrm{w}]$ can occur in the coda position.

Linguists have debated for decades on how many vowels there are in the Mandarin phonemic inventory (Cheng 1973, Lin 1989, Wang 1993, Duanmu 2000). Table 2 presents the co-occurrence constraints on consonants and high vowels in the syllable structure $\mathrm{CV}$. The vowels [i] and [u] only occur in CV syllables. For the high vowels, the distribution of [y] is much more restricted than other vowels. The vowels [i], [i] , and [u] are in complementary distribution. [i] only occurs after $\left[\mathrm{s}, \mathrm{ts}, \mathrm{ts}{ }^{\mathrm{h}}\right] ;$ [u] only appears after [s, ts, ts $\left.\mathrm{s}^{\mathrm{h}}, \mathrm{z}\right]$ and [i] elsewhere. The minimal set [i, y, u] can be found after [n] or [1]. The question is, do Mandarin speakers consider [i], [i], and [u] as the same vowel or distinct vowels?

Table 2. Occurrence of consonants and high vowels

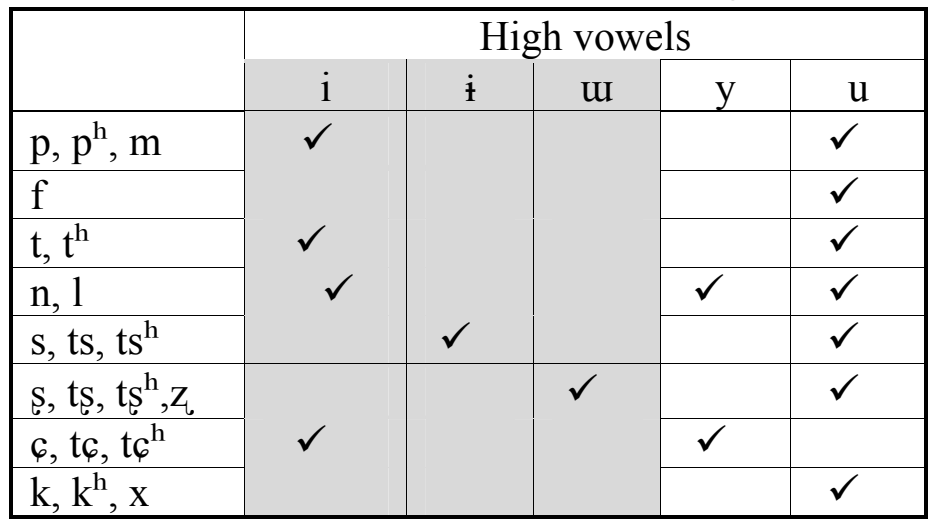

Table 3 shows the distribution of mid vowels in different syllable structures in the surface forms. The vowel [e] may only be preceded by one of the glides $[j, y]$

\footnotetext{
${ }^{1}$ In this paper, $\mathrm{C}$ is the abbreviation for consonant; $\mathrm{G}$ is for glide; $\mathrm{V}$ is for the nucleus vowel and $\mathrm{N}$ is for nasals. Also, the phonological input of sounds will be annotated with //; the phonological output of sounds will be presented with [ ]; the pinyin transcription will be shown with ',
} 
in $\mathrm{CGV}(\mathrm{N})$ syllables; the vowel [ə] exists in $\mathrm{CV}(\mathrm{N})$ or $\mathrm{CGV}(\mathrm{N})$ syllables when $\mathrm{G}$ is the glide $[\mathrm{w}]$. The vowel [e] is in complementary distribution with the vowels $[ə]$ and $[0]$.

Table 3. Occurrence of syllable structures and mid vowels

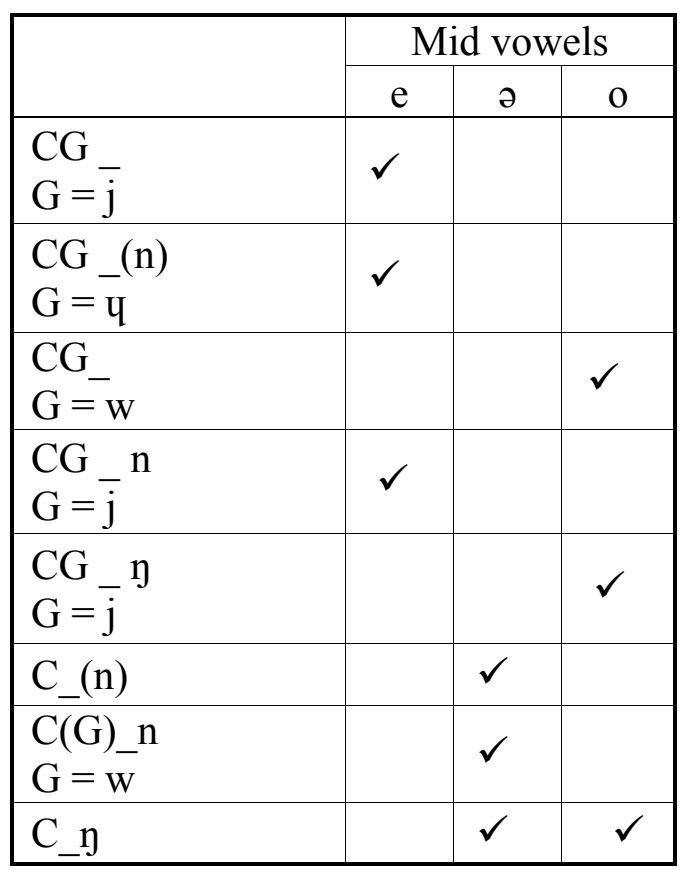

With regard to low vowels, the back low vowel [a] occurs in an open syllable or before the coda $[\mathrm{y}]$. The front low vowel [a] only occurs before the velar nasal [n], as shown in Table 4.

Table 4. Occurrence of syllable structures and low vowels

\begin{tabular}{|l|c|c|}
\hline \multirow{2}{*}{\multicolumn{2}{|c|}{}} & \multicolumn{2}{|c|}{ Low vowels } \\
\hline C__ & & a \\
\hline C(G)_n & $\checkmark$ & \\
\hline C(G)_y & & $\checkmark$ \\
\hline
\end{tabular}

Complementary distribution is only one way to group sounds as allophones of a phoneme. In English, [h] does not occur in word-final position, while [y] does not appear in the word-initial position. Hence, they are in complementary distribution. However, $[\mathrm{h}]$ is not considered an allophone of $[\mathrm{g}]$ because they do not 
Chen-huei Wu and Chilin Shih

sound similar. In this paper, vowel categories will be examined in terms of acoustics and articulation.

\section{How Many Vowels in Mandarin?}

In this section, we will review how the vowels in Mandarin have been categorized from different perspectives, including the transcription systems, phonological categories, phonetic measurements and articulatory investigations in the previous literature. The transcription systems reflect how native speakers have been taught to acquire their sound system explicitly. The phonological categories present the abstract representation of vowels. Phonetic measurements provide the acoustic values of vowels and the articulatiory investigation describes the physiological and kinematic movements of vowels. Different approaches sketch different dimensions of Mandarin vowels and help us to understand them.

\subsection{Transcription Systems}

The Chinese writing system uses symbols known as Chinese characters. There are thousands of characters in common usage, and tens of thousands if rare characters and archaic forms are included. Many transcription systems were developed to annotate pronunciations as a study aid in school systems. Two such systems are used widely today. Zhuyin is used in Taiwan, and Pinyin in used in China. Zhuyin uses distinct symbols to represent 21 onset consonants, 3 glides and 13 rhymes. Two of the rhymes ([i, u] $)$ are not assigned distinct symbols, and the 3 glides are also used to represent vowels. In sum, this system recognizes 18 rhymes, including the two signs that are not written. Pinyin is a Romanization system that uses Roman letters to represent onset consonants, glides, vowels and nasal codas. In the Pinyin system, the vowels in Mandarin are presented by six symbols 'a, e, i, o, $\mathrm{u}$, ü'. These two transcription systems give us the range of the possible number of surface vowels in Mandarin: anywhere between 6 and 18 .

\subsection{Phonological Categories of Mandarin Vowels}

By "surface vowels", we mean the phonological output. It is not the same as the phonological input, or the underlying system. In Mandarin tonal phonology, there are four tones in the phonological input and still four tones in the output after tone sandhi rules (Shih 2008). Unlike Mandarin tones, it is not clear how many Mandarin vowels exist in the underlying representation and the surface forms. Phonologically, many vowels are predictable from context. For example, the rewrite rule in (1) expresses that the back low vowel /a/ will become the front low vowel /a/. $/ \mathrm{a} /$ in the rewrite rule is the phonological input or the phonological underlying representation, while /a/ is the phonological output or the surface form.

\section{(1) $\quad \mathrm{a} \rightarrow \mathrm{a} / \ldots \mathrm{n}$}

According to various theoretical approaches, the number of Mandarin vowels ranges from two to six in the underlying representation and nine to fifteen in the 


\section{Mandarin Vowels Revisited}

surface forms (Cheng 1973, Lin 1989, Wang 1993, Duanmu 2000). Cheng (1973) proposes six underlying vowels in Mandarin based on whether a vowel can occur in CV syllables, as opposed to surface representations of twelve vowels. Based on underspecification theory, Lin (1989) presents a five-vowel system and surface manifestations of eleven vowels. The eleven vowels are created as the result of assimilation processes. One of the most extreme analyses is Wang (1993), who suggests there are only two vowels in Mandarin. This is done by treating high vowels as glides underlyingly. Duanmu (2000) suggests that Chinese has five vowels phonemically and nine forms in the surface representation. His analysis is based on evidence of rhyming and the distribution of vowels in the syllable structure GVX.

\subsection{Phonetic Categories of Mandarin Vowels}

A central issue within speech technologies such as text-to-speech synthesis (hereafter TTS) is the naturalness of the system, which requires an optimal collection of acoustic inventory and an understanding of how the pronunciations vary in context. Howie (1970) was the first study to obtain a systematic acoustic description of Mandarin vowels, including phonemes and allophones in the occurrence of the four tones.

Shih (1995) investigates the acoustic properties of Mandarin vowels for the purpose of achieving naturalness in TTS. In Shih (1995), the findings show the following: (1) For diphthongs, the vowel nucleus is typically different from the corresponding monophthongs, with the exception of the diphthong [ow]. That is, [a] in [aj], [aw] and [e] in [ej] are different from monophthongs [a] and [e], respectively. Diphthongs are only similar to the corresponding monophthongs at the beginning $20 \%$ portion. (2) Coarticulation effects on schwa [ə] and the low vowel [a] are observed. The effects of the following nasals [n, $y$ ] in [ən] and [əy] and the preceding glides $[\mathrm{j}, \mathrm{w}]$ in [ja] and [wa] are expected and consistent with the anticipated tongue position of the sounds in context.

\subsection{Articulatory Studies of Mandarin Vowels}

One major articulatory study of Mandarin vowels is reviewed here. In an Electromagnetic Midsagittal Articulography study (Torng 2000), 24 words consisting of five Mandarin vowels [i, y, u, a, o] with four tones were measured. The results are summarized as follows: (a) For the tongue body position, the high vowel [i] has the highest absolute tongue height followed by [y], [u], [o], [a], as expected. (b) For jaw position, vowels [u, y] have high jaw positions and the vowel [a] has a low jaw position. Unexpectedly, the mid vowel [o] has a jaw position as high as the high vowel $[\mathrm{u}]$ and the high vowel [i] has a lower jaw position than $[\mathrm{u}, \mathrm{y}, \mathrm{o}]$. (c) Vowels $[y, u, o]$ have stronger lower lip protrusion and vowels [i, a] have weaker lip protrusion. As expected, vowels $[\mathrm{y}, \mathrm{u}]$ have a smaller lip aperture and vowels $[\mathrm{i}, \mathrm{o}, \mathrm{a}]$ have a larger lip aperture. (d) The tongue body position is determined by the jaw position since the tongue rests on the jaw. One exception is the 
vowel [o]. The derived tongue position shows that the vowel /o/ has a lower tongue position than the vowel /a/.

Consideration of transcription systems, phonological categories, and phonetic properties have not answered the question concerning how many vowels there are in Mandarin. Basically, there is no agreement in the previous research. The goal of this study is to investigate the phonological output of vowels in Mandarin in terms of the articulatory demonstration of the positions of the tongue and lips, as well as the corresponding acoustic results of the articulatory data. Further, we examine all vowels in context, but we are not limited to the five vowels [i, y, u, a, o] as in the previous articulatory study (Torng 2000).

\section{Methodology}

\subsection{Materials}

The data were collected from one native Mandarin speaker from Taiwan, who produced all possible Mandarin syllables including vowels with tone 1. Each word was read in a frame sentence, /ts $r \mathrm{k}$ _ _ tsi/ 'This ___ word', to avoid the lengthening effect of producing the test words in the final position of a sentence. Stimuli were presented in traditional Chinese characters. A few syllables do not occur with tone 1 . Therefore, no Chinese characters can represent these sounds. In those cases, Zhu-yin symbols were used to annotate the sounds.

\subsection{Procedure}

The data were obtained from a Carstens 3D-Articulograph AG500. This apparatus consists of the EMA cube with six transmitter coils generating magnetic fields at different frequencies at defined orientations, 12 sensors and channels, a computer with an automatic calibration unit, real time display, head movement correction systems, and a receiver. Six transmitter coils generate alternating magnetic fields at different frequencies. Sensors, which consist of small coils, are fixed onto the articulators of the subject. The alternating currents induced by the alternating magnetic fields have different strengths as a function of the distance and the angle of the sensor to the respective transmitter coil (Carstens Medixinelektronik 2006). A microphone mounted on a microphone stand and extended on a boom stick is connected to the audio box, transferring the speech signal to the synchronizer. All articulatory and acoustic data are acquired and synchronized simultaneously.

The participant was seated in a wooden chair and their head was positioned in the center of the EMA cube. The filled circles in Figure 1 schematize the approximate locations of the sensors used in this experiment. Sensors were mounted on the tongue tip (TT, $1 \mathrm{~cm}$ behind apex), the tongue body (TM, $1 \mathrm{~cm}$ behind the tongue tip sensor) and the tongue back (TB, $1 \mathrm{~cm}$ behind the tongue center sensor), as well as on the lower incisor (LI), the upper lip (UL), the lower lip (LL), and the right and left corners of the lip. Other sensors were attached to the bridge of the nose and the left and right tragi as reference points to normalize head movement. During the experiment, the sensor on TB fell out in the recording process and the data from TB were excluded from the analysis. 


\section{Mandarin Vowels Revisited}

After obtaining the data, digitalizing of the reference point signal and head movement correction were carried out. The acoustic data were analyzed with WAVES+/ESPS software. Auditory judgments of vowel quality were made to verify correct production for acoustic analyses. With 406 possible syllables in Mandarin, 387 out of 406 were correctly produced. The target words were elicited from the speech strings and were segmented manually by the author. Possible measurement error with the EMA system and formant tracking with WAVES+ were examined and eliminated from the data analysis. The formant frequency and articulatory data at the mid point of the vowel duration were used to do the analysis with R statistical software.

Figure 1. Schematic view with seven sensors on the tongue and lips as well as a reference point on the bridge of the nose.

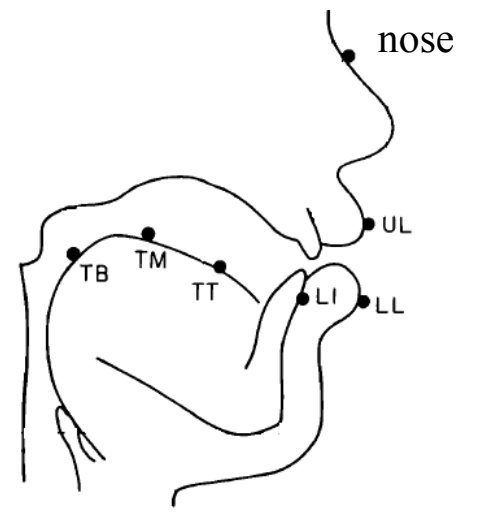

TB: Tongue Back

TM: Tongue body

TT: Tongue Tip

LI: Lower Incisor

UL: Upper Lip

LL: Lower Lip

\section{Analysis of the Data and Discussion}

Figure 2 shows the mean values at the points of the tongue body and the tongue tip in the $\mathrm{X}$ (anterior and posterior) and $\mathrm{Z}$ (superior and inferior) dimensions for vowels [i, y, u, e, ə, o, a]. For the tongue body height, [i] has the highest tongue body position, followed by vowels $[\mathrm{y}],[\mathrm{e}],[\mathrm{u}],[\mathrm{e}],[\mathrm{a}]$ and $[\mathrm{o}]$. As for the tongue tip height, [y] has the highest tongue tip position, followed by vowels [i], [e], [ə], [u], [o], [a]. This result is similar with previous studies (Torng 2000) with the unexpected exception that the vowel [a] has a higher tongue body position than the vowel [o]. This observation is also found in Torng (2000). As for the anterior and posterior dimension, the vowels $[\mathrm{i}, \mathrm{y}, \mathrm{e}]$ have the greatest anterior tongue body position, followed by the vowels $[\mathrm{u}] /[\mathrm{\partial}] /[\mathrm{a}]$ and then $[\mathrm{o}]$. The vowel $[\mathrm{y}]$ has the greatest anterior tongue tip position, followed by the vowels $[\mathrm{i}] /[\mathrm{e}],[\mathrm{u}] /[\mathrm{a}]$, and then $[0] /[0]$.

The formant analysis of the articulatory data in Figure 2 is illustrated in Figure 3 , which shows the formant space of the vowels plotted by the mean values of the first formant (F1) and the second formant (F2) at the midpoint. As expected, the vowel [i] has the lowest $\mathrm{F} 1$, followed by $[\mathrm{y}],[\mathrm{u}],[\mathrm{e}],[\mathrm{\rho}] /[\mathrm{o}]$ and [a]. Also, the vowel [i] has the highest F2, followed by vowels [y], [e], [ə]/[a], [o] and [u]. 


\section{Chen-huei Wu and Chilin Shih}

Figures 2 and 3. Tongue positions (left) and formant chart (right) of Mandarin vowels
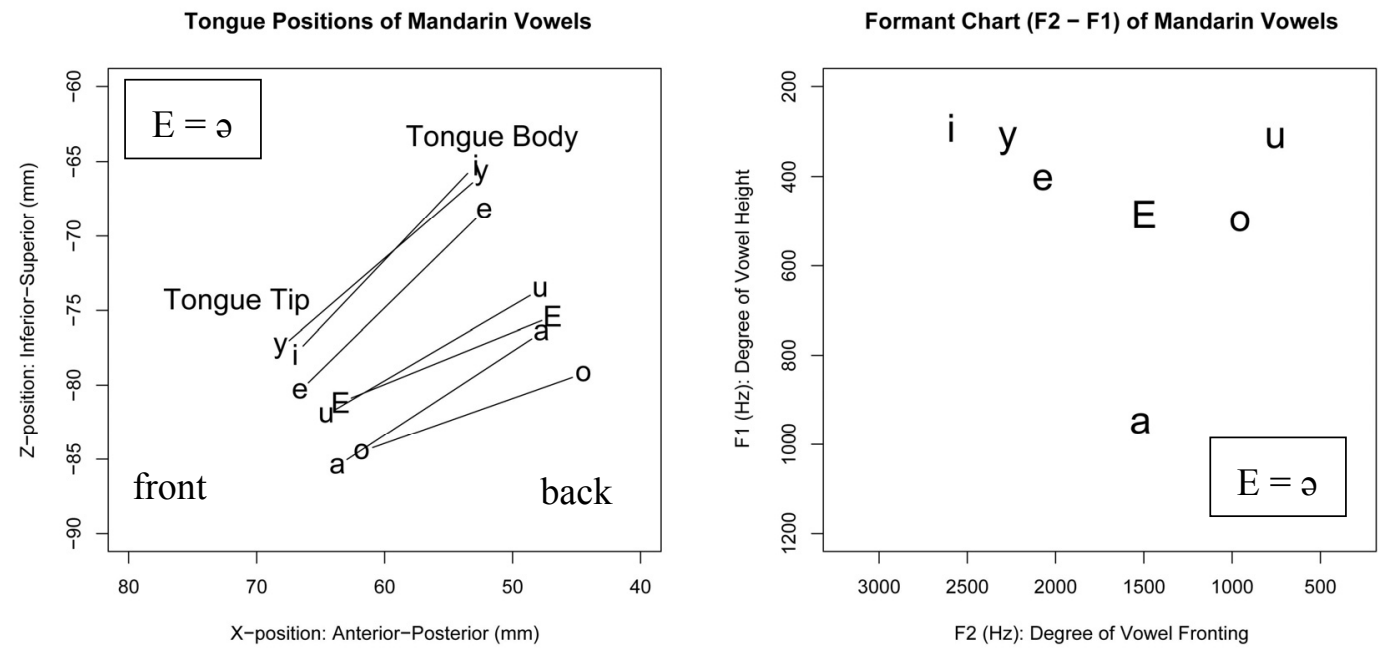

Figures 4 and 5. Tongue body position of [i] (left) and [a] (right), with variants
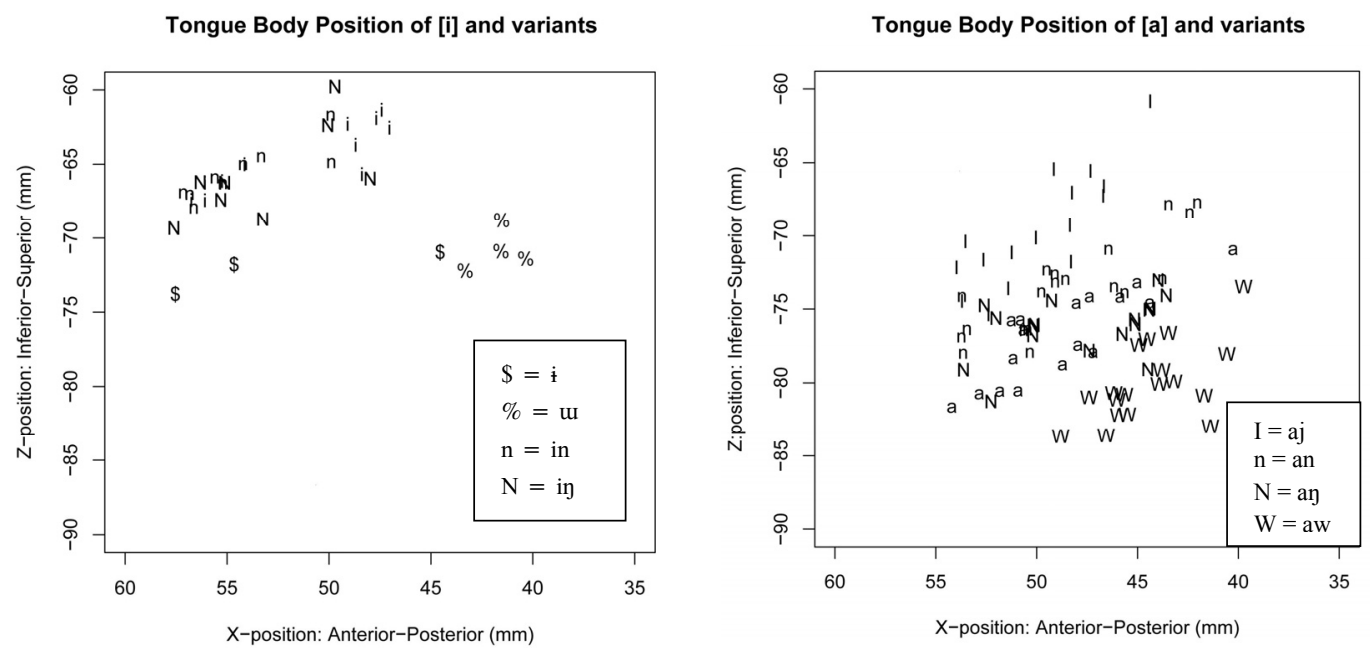

Figures 4 and 5 demonstrate the mean values at the points of the tongue body in the $\mathrm{X}$ (anterior and posterior) and $\mathrm{Z}$ (superior and inferior) dimensions of the vowels [i] and [a] and their variants. The physical distance of the tongue positions in the vowel [a] is greater than the case of the vowel [i] in [in] and [ii]. The [a] in [an], [ay], [aj] and [aw] are fairly distinguishable articulatorily and acoustically. This finding is similar to Perkell and Nelson (1985), which shows the variations in production vowels [i] and [a] on the tongue, suggesting the vowel [i] has a relatively small size of distribution compared with the vowel [a].

The discrepancies between the articulatory and the acoustic data can be explained. Traditionally, the formant values of vowels are usually related to articulatory descriptions of vowels. That is, F1 represents the vowel height as the tongue body height and F2 represents the backness of vowels as the anterior and 


\section{Mandarin Vowels Revisited}

posterior positions of the tongue. However, the formant values of vowels are determined by the position of the maximum constriction of the vocal tract, which affects the length and the cross-sectional area of the front and back cavities (tubes) in the multi-tube models of vowel production (Stevens and House 1955, Fant 1960). Ladefoged (1975) has pointed out that the position of the highest point of the tongue is not a valid indicator of vowel quality. The term 'vowel height' refers to auditory quality that is specified in acoustic properties rather than in articulatory positions.

In the current data, the analysis of the articulatory positions of the tongue and formant frequencies reveals discrepancies between traditional phonetic descriptions of vowels and the actual tongue positions. In terms of the acoustic vowel height, the vowel [e] is a mid vowel and the vowel [u] is a high vowel (low F1). However, the vowel [e] has a higher tongue position than the vowel [u]. Similarly, the vowel [a] is a low vowel (high F1) and the vowel [o] is a mid vowel, while the vowel [a] has a higher tongue position than the vowel [o]. In other words, the vowels [e] and [a] have higher tongue body positions than the vowels $[\mathrm{u}]$ and $[\mathrm{o}]$, respectively. In terms of the backness, the vowels $[\mathrm{u}]$ and [o] have a more posterior tongue body position than the vowel [e]. The tongue body position in Figure 3 does not indicate the place of maximum constriction of the vocal tract. Since we know that vowels $[\mathrm{u}]$ and $[\mathrm{o}]$ have constrictions both at the lips and at the soft palatal area, the retracted tongue body position and the lip rounding of the vowels $[\mathrm{u}]$ and [o] maintain lower F1 than the vowels [e] and [a], respectively.

In phonological or phonetic representation, distinctive features are used to classify speech sounds in terms of acoustic cues (Jakobson et al. 1952). Later reformulation of distinctive features by Chomsky and Halle (1968) reflects more articulatory properties than acoustic and perceptual correlates. Others may have suggested that some features might have better-defined auditory correlates than articulatory correlates and vice versa.

Daniel Jones (1956) was the first to draw the vowel space, the cardinal vowels, to visualize tongue positions in articulation of vowels. Jones envisioned cardinal vowels to be of equal distance to one another. The articulatory data in the current study show that the tongue positions are much more complicated than this view. However, it has been discussed whether the distinctive features describing vowels should be defined in terms of tongue height, in terms of acoustic properties, or in terms of perceptual distance. Regarding examining the articulatory correlates of vowel categories, Stevens (1975) has suggested that different patterns of contact between the tongue and maxilla may define vowel height categories. Lindau (1975) has pointed out that there is evidence against the traditional concepts of vowel height as the height of the highest point on the tongue, particularly for back vowels. Similarly, Ladefoged (1975) demonstrates that the tongue height of back vowels bears very little relation to vowel height. A different approach proposes that vowels can be categorized by the place of articulation (Wood 1979, Hasegawa-Johnson et al. 2003). Based on the constriction of the vocal tract, /i, e, I, $\varepsilon /$ are classified as palatal vowels, /u, $\mathrm{u} /$ are velar vowels, /o, o/ 
are uvular vowels, and /a, a, $\Lambda, æ /$ are pharyngeal vowels. The place of articulation more or less corresponds to the backness of the vowels.

In the current study, the data show that the vowel height in terms of the acoustic measurements is not equivalent to the tongue height in terms of articulatory positions. Vowel height better reflects the acoustic properties than articulatory movements. In quantal theory (Stevens 1972, 1989), the relation between the articulatory parameter and acoustic output is not linear. Linguistically, contrastive sounds involve differences between quantal regions. In the analysis of Mandarin vowels, the tongue positions of the monophthong [i] and [i] in [in] are similar, but slightly different from [i] in [ii]. Tongue positions and formant values of the vowels [i] and [u] distinguish them from [i] more than the [i] influenced by coda nasals. The question is whether they belong to the same or different categories due to the contrast quantal regions.

The problem of phonological categories of the vowels in different contexts is if native speakers perceive them as having the same quality and belonging to the same groups. It may not be controversial to classify the monophthong [i] and variants of [i] in [in] and [ii] in the same categories due to the small physical distance of the tongue positions and similar formant values. Due to the complementary distribution of the vowels [i], [i] and $[\mathrm{w}],[\mathrm{i}]$ and $[\mathrm{w}]$ are treated, in the previous literature, as variants of the vowel [i] in the phonological input. However, it is possible that vowels [i], [i] and [u] may be treated as different vowel/phonetic/perceptual categories based on the greater differences of acoustic properties and articulatory positions from the vowel [i]. Furthermore, the articulatory and acoustic properties of the monophthong [a] and variants as [a] in [an], [ay], [aj], and [aw] are fairly different. It is not clear whether the discrepancies in [a] lead to different categories.

Vowel perception may be affected by both articulatory and perceptual factors. One way to test this issue is to examine native listeners' perceptual judgments of the vowels in different contexts. A perceptual experiment of vowel classification will be conducted to answer this question in future studies.

\section{Conclusion}

In this paper, we reexamine the categories of Mandarin vowels in terms of articulatory positions and acoustic properties. We review this issue from the perspectives of the influences from the transcription systems, phonological representation, acoustic-phonetic measurements, as well as articulatory properties. We investigate the production of Mandarin vowels with the EMA system in terms of acoustics and articulation.

The analysis of the articulatory positions of the tongue and formant frequency reveals some discrepancies between traditional phonetic descriptions of vowels and the actual tongue positions. The data in the present study shows that the changes in articulation do not necessarily change the acoustic output. For instance, the tongue height does not completely correspond to the vowel height in formant values. In addition, the vowel [a] is influenced by a wider range in 


\section{Mandarin Vowels Revisited}

different contexts than the vowel [i]. This topic is important not only to understand the reality of vowels in Mandarin, but also to help the second language acquisition of the sound system, and speech recognition. A perception study of vowel categorization will be carried out and the relationships among articulation, acoustics and perception will be explored in future studies.

\section{Acknowledgements}

This work is supported in part by NSF IIS-0623805, NSF IIS-0534133, and a Critical Research Initiatives Grant from UIUC. We thank Mark HasegawaJohnson and Torrey Locks for their valuable comments. The views and conclusions are those of the authors and do not necessarily reflect the views of the National Science Foundation and UIUC.

\section{References}

Carstens Medixinelektronik GmbH. 2006. AG 500 Manual. http://www.ag500.de/ ida/AG500_manual.pdf

Cheng, Chin-chuan. 1973. A synchronic phonology of Mandarin Chinese. Monographs on Linguistic Analysis 4. The Hague: Mouton.

Chomsky, Noam, and Morris Halle. 1968. The sound pattern of English. New York: Harper and Row.

Duanmu, San. 2000. The phonology of Standard Chinese. Oxford \& New York: Oxford University Press.

Fant, Gunnar. 1960. Acoustic theory of speech production. The Hague: Mouton.

Hasegawa-Johnson, Mark, Shamala Pizza, Abeer Alwan, Jul Cha, and Katherine Haker. 2003. Vowel category dependence of the relationship between palate height, tongue height, and oral area. Journal of Speech, Language, and Hearing Research 46.3: 738-753.

Howie, John Marshall. 1970. The vowels and tones of Mandarin Chinese: Acoustical measurements and experiments. Ph.D. diss., Indiana University.

Jakobson, Roman, Gunnar Fant, and Morris Halle. 1952. Preliminaries to speech analysis: The distinctive features and their correlates. Cambridge, MA: MIT Press.

Jones, Daniel. 1956. An outline of English phonetics. $8^{\text {th }}$ ed. Cambridge: Heffer.

Ladefoged, Peter. 1975. A course in phonetics. $1^{\text {st }}$ ed. New York: Harcourt, Brace Jovanovich.

Lin, Yen-hwei. 1989. Autosegmental treatment of segmental processes in Chinese phonology. Ph.D. diss., University of Texas, Austin.

Lindau, Mona. 1975. Features for vowels. UCLA Working Papers in Phonetics 30. Department of Linguistics, University of California, Los Angeles.

Perkell, S. Joseph, and Winston L. Nelson. 1985. Variability in production of the vowels /i/ and /a/. Journal of the Acoustical Society of America 77(5): 18891895. 
Shih, Chilin. 1995. Study of vowel variations for a Mandarin speech synthesizer. Proceedings of EUROSPEECH-1995, 1807-1811. Madrid, Spain: European Speech Communication Association.

Shih, Chilin. 2008. Linking phonology and phonetics: An implementation model of tones. In Yuchau E. Hsiao, Hui-chuan Hsu, Lian-Hee Wee, and Dah-an Ho (eds.), Interfaces in Chinese phonology: Festschrift in honor of Matthew Y. Chen on his 70th birthday, 99-120. Language and linguistics monograph series W8. Taipei: Institute of Linguistics, Academia Sinica.

Stevens, Kenneth N. 1972. The quantal nature of speech: Evidence from articulatory-acoustic data. In P.B. Denes and David, E. E., eds., Human communication, a unified view, 51-66. New York and London: McGraw-Hill.

Stevens, Kenneth N. 1975. Quantal configuration of vowels. Journal of the Acoustical Society of America 57, S70 (A).

Stevens, Kenneth N. 1989. On the quantal nature of speech. Journal of Phonetics 17: 3-45.

Stevens, Kenneth N., and Arthur S. House. 1955. Development of a quantitative model of vowel articulation. Journal of the Acoustical Society of America 27: 484-493.

Torng, Pao-chuan. 2000. Supralaryngeal articulator movement in Mandarin Chinese tonal production. Ph.D. diss., University of Illinois, UrbanaChampaign.

Wang, Jenny Zhijie. 1993. The geometry of segmental gestures in Beijing Mandarin. Ph.D. diss., University of Delaware, Newark.

Wood, Sidney. 1979. A radiographic analysis of constriction locations for vowels. Journal of Phonetics 7: 25-43.

Chen-huei $\mathrm{Wu}$

Chilin Shih

University of Illinois, Urbana-Champaign

Department of Linguistics

4080 Foreign Language Building

707 S Mathews Avenue, MC-168

Urbana, IL 61801

wu9@illinois.edu

cls@illinois.edu 